\title{
THE RELATION OF ANOXEMIA TO THE TYPE OF BREATHING IN PNEUMONIA
}

\section{A Study of Respiration by Means of a Body Plethysmograph}

By CARL A. L. BINGER AND JOHN STAIGE DAVIS, JR.

(From the Hospital of The Rockefeller Institute for Medical Research, New York)

(Received for publication July 17,1928 )

\section{INTRODUCTION}

The type of breathing in pneumonia has certain fairly well marked characteristics, which distinguish it from other disturbances of respiration. There is, as a rule, an increased respiratory rate with a decrease in the depth of each breath. This limitation in respiratory excursion is in most instances accompanied by more or less severe pleuritic pain. To what extent the pain is responsible for the shallow breathing or for the so-called expiratory grunt is not clear, though it is fairly certain that it is not wholly accountable for either. With the exception of the pleuritic pain, the patient suffering from pneumonia seems extraordinarily unconcerned with his embarrassed breathing. It is a matter of common observation that patients with pneumonia do not complain of breathlessness even when they are well enough to enumerate their symptoms. Nor do they, except when in extremis, appear to make an extra effort to get their breaths, as do individuals suffering from heart failure or from severe uncompensated acidosis or asthma. Furthermore, most of them are apparently quite content to lie flat in bed. These facts suggest that the respiratory mechanism in pneumonia, although embarrassed, is operating fairly efficiently at a new level. Consistent with this suggestion have been the findings with respect to the acid-base balance in pneumonia. There appears to be no evidence for the existence of an acidosis. Indeed, the blood in only an occasional case shows any deviation from what is considered to be a normal zone delimited by the $\mathrm{pH}$ and $\mathrm{CO}_{2}$ tension lines (1). When such a deviation occurs it exists in the direction of a slight compensated alkalosis rather than of an acidosis. The maintenance of 
these physiological constants at their normal levels shows that the respiratory function is, in fact, in fair working order. This statement must, however, be qualified, since the other chief function of the respiration - the supply of oxgen to the tissues-is impaired. No actual measurement of the oxygen tension in the tissues has been made, nor of the tension in the arterial blood of pneumonia patients. The oxygen content and capacity of the arterial blood is, however, readily measurable, and from these figures one can obtain an estimate of the efficiency of the respiratory mechanism.

It is probably not inaccurate to say that almost every case of pneumonia shows at some time a deficiency in the oxygen saturation of the arterial blood (2). The fact that there may be oxygen want and still no carbon dioxide retention or shift in the neutrality regulation of the blood is explicable on the basis of the different physical properties of $\mathrm{O}_{2}$ and $\mathrm{CO}_{2}$ and the different manner in which they combine with hemoglobin. This subject has been fully discussed by Haldane (3).

Lundsgaard and Van Slyke (4) have attempted to analyze the various factors which contribute to the presence of cyanosis. The origin of cyanosis in pneumonia, it seems safe to state, is respiratory rather than circulatory. It is, however, by no means clear why the blood is incompletely oxygenated in the lungs. Several explanations have been offered for this, viz., unequal expansion of the lungs (5); rapid and shallow breathing (6); diminished lung volume (7); intra-alveolar exudate (8); decreased oxygen diffusion resulting from toxic injury of alveolar walls (9); passage of blood through unaerated channels in the lung (10). It has, moreover, been shown experimentally that an increased rate of blood flow through the lungs may prevent the hemoglobin from taking up its normal load of oxygen (11). Whether this last actually plays a rôle in pneumonia is, to be sure, doubtful, but there seems little doubt that some or all of the other factors may combine to prevent the complete reoxygenation of the blood in its passage through the lungs. Plausible as these explanations may be, the actual proof of their influence and the proper weighting of their importance remains difficult, if not impossible. One can never know the true state of the parenchymatous and vascular lesion in the lung at the time of the arterial oxygen analysis. The relationship of these two must therefore remain a matter of inference and conjecture. 
It is far easier to measure the changes in the type of breathing and to correlate these with changes in the oxygen saturation of the arterial blood. One should not, however, give too much weight to such a correlation, because there will remain other factors not accessible by quantitative methods, but no less important. It is more profitable for the present to state the relationships observed than to give undue weight to measurements simply because they represent the only data available.

\section{METHODS}

We have recently described a body plethysmograph for the study of respiratory movements in human beings (12). The measurements made in the present work were obtained by placing the patients in the plethysmograph. This we believe to be a more accurate method of recording respirations in the presence of already embarrassed breathing than is possible with the use of masks or mouth pieces even though valves of minimal resistance are available. We have frequently observed that profound changes in breathing result from the application of these various devices to the patient's head. The plethysmograph has the added advantage of permitting a record of the observations while the subject is ignorant of the fact that his respirations are being studied. The patients were allowed to remain in the box for 10 to 20 minutes before observations were begun. During this time any feeling of apprehension seemed to pass off and the subject frequently dozed. It was impossible to secure data on patients who were desperately ill as it seemed inadvisable to move such individuals to the laboratory.

A graphic tracing (fig. 1) was obtained for a period varying in length from onehalf to one hour. No preliminary fast was required, but in most instances observations were made 2 or 3 hours after meals. During each tracing, the plethysmograph and recording apparatus was tested for leaks. At the close of the period of observation, the subject was urged to take the deepest breath he could for the purpose of measuring his complementary air and vital capacity. An arterial puncture was then performed, usually of the femoral artery. The blood was analysed for its oxygen content and capacity by the method of Van Slyke and Neill (13). The data thus acquired give us information about the rate and depth of respiration, the relative duration of inspiratory and expiratory phases, the vital capacity of the lungs and the oxygen content, capacity and per cent saturation of the arterial blood. These facts so simply acquired in normal individuals are far from easily obtained from patients suffering from pneumonia. Great care must be taken that the procedures should not fatigue the patient and jeopardize his chances of recovery. Not infrequently we have had to omit from the series 


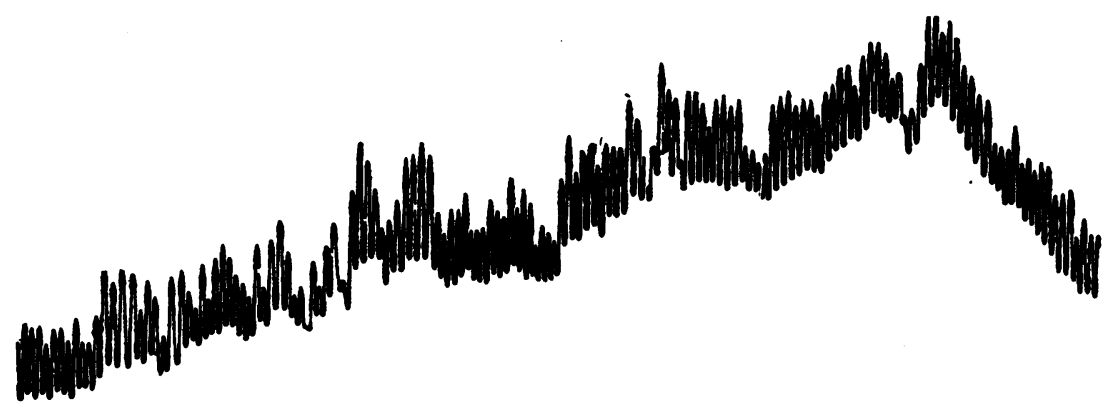

(a)

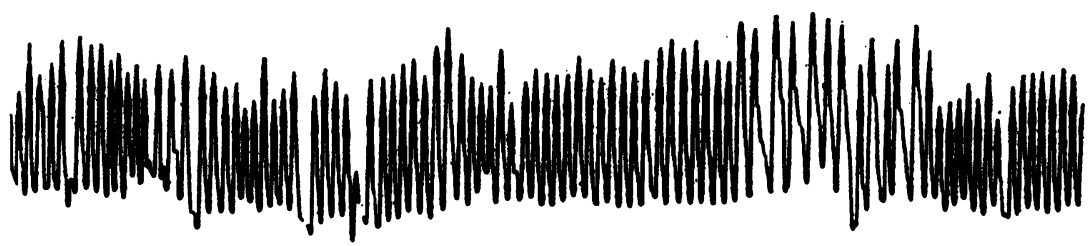

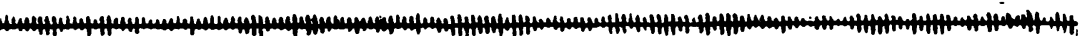

(b)

Fig. 1. (a) Plethysmographic Tracing from Case 10, Made on the Fourth Day after Onset of Illness; (b) Plethysmographic Tracing from Same Patient Made during Convalescence, on Twenty-Fifth Day AFTER ONSET OF ILLNESS

The upper line was drawn by the work-adder signal lever. Interval between signal lever marks equals 12.34 liters. The lower line represents time in 2 second intervals. 
individuals best suited for the purposes of the study because their general condition did not seem to warrant subjecting them to any unnecessary disturbance.

The series studied consists of 17 patients, all suffering from acute pneumonia, in the Hospital of The Rockefeller Institute (table 1). The general plan was to study the patient during the acute stage of his disease and again during convalescence. The variegated nature of the material makes a grouping of data of dubious value. More is to be learned from a consideration of the changes occurring in the individual patients.

In general it may be said that the abnormalities in breathing consist in rapid and shallow respiratory movements resulting in an increased minute volume of pulmonary ventilation. Associated with this, there is a shortening of the duration of both respiratory phases, more particularly of the expiratory. There is, of course, a marked diminution of the vital capacity of the lungs. All these changes are closely related to the condition of anoxemia as expressed by the oxygen saturation of the arterial blood. To what extent abnormalities in breathing are responsible for oxygen want, or whether anoxemia in turn gives rise to the disturbances of respiration it is the chief object of this investigation to discover.

\section{EXPERIMENTAL}

Of 11 patients in whom observations were made both during the acute stage of the disease and during convalescence, 8 showed a definite correlation between rapid and shallow breathing and anoxemia. By this we mean that when restored to health the patients not only showed an increase in the per cent saturation of the arterial blood, but slower and deeper breathing and a diminution in the minute volume of pulmonary ventilation. From this fact alone no conclusion can be drawn as to a possible causal relationship between rapid and shallow breathing and anoxemia. The two may simply occur coincidentally or be related to a common cause.

No constant relationship can be shown to exist between the per cent change in respiration and the per cent change in saturation. For example, case 1 showed an increase in tidal air of 76 per cent during convalescence with an 11 per cent rise in saturation, while case 2 


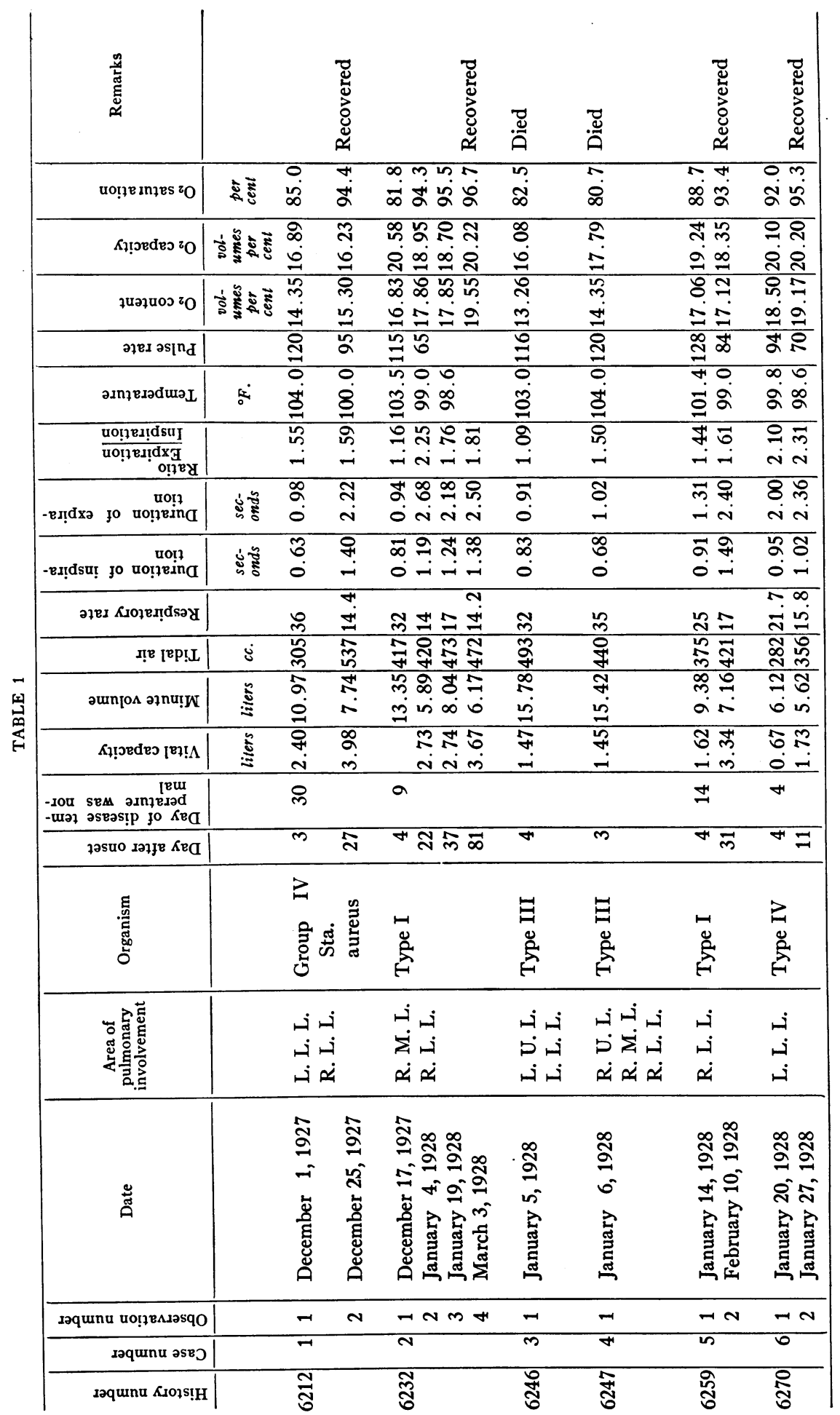




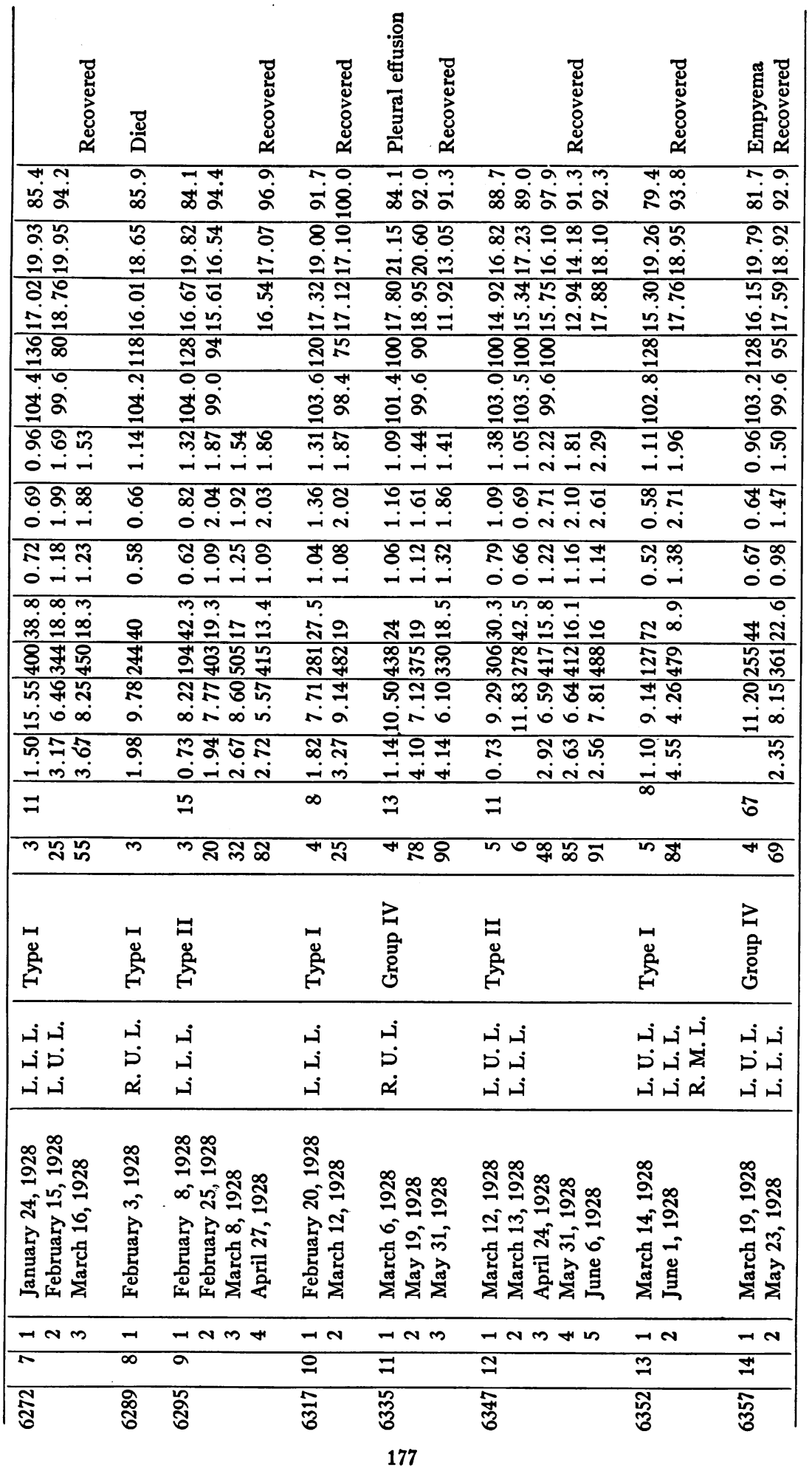




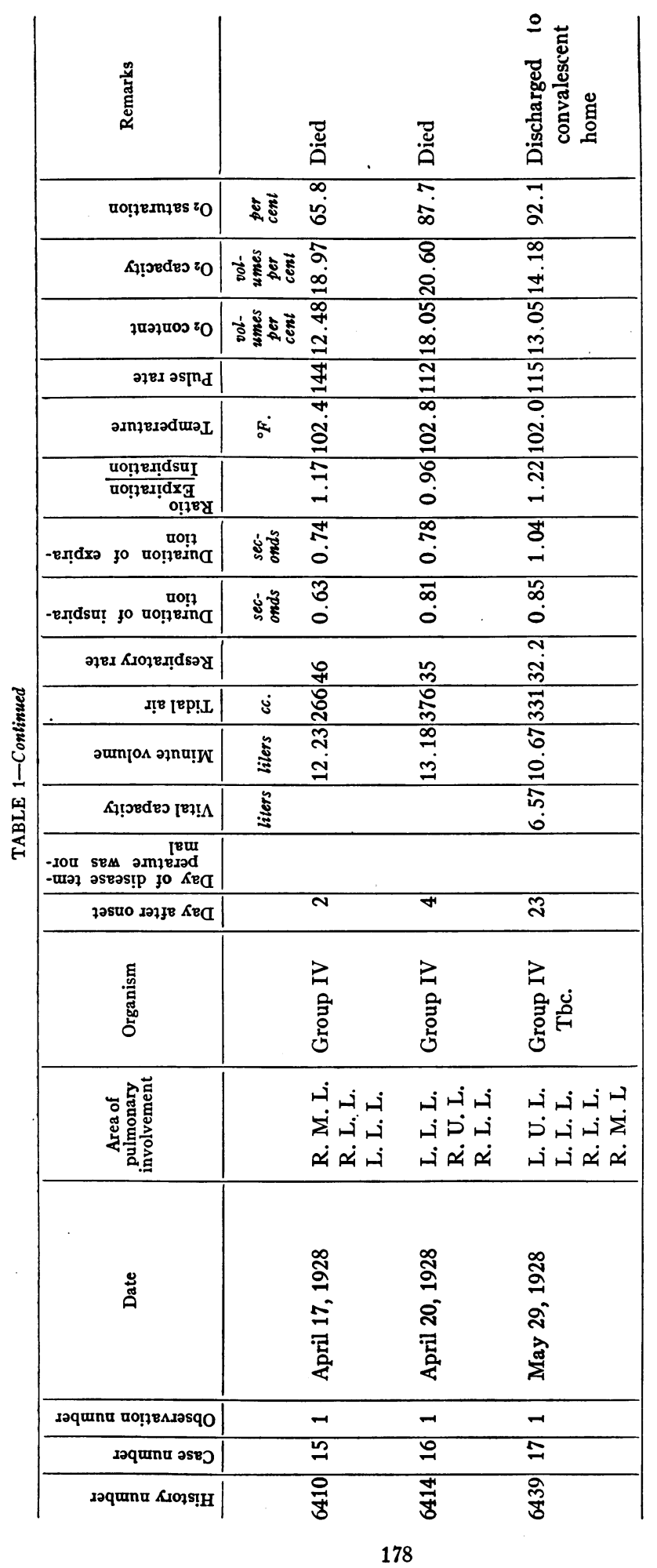


showed a greater rise in saturation, over 15 per cent, with practically no change in tidal air. In both cases, however, there was an approximately equal reduction in respiratory rate. In case 5 the tidal air increased by 12 per cent with a 5 per cent rise in saturation while case 6 showed a 26 per cent gain in depth but only a 3 per cent gain in saturation. Cases 7 and 11 both showed an actual diminution in tidal air with convalescence though in the first the arterial saturation rose from 85.4 to 94.2 and in the second from 84.1 to 92.0 per cent.

In case 9 the gain in saturation was 12 per cent which was less than the gain in case 14 in spite of the fact that the former patient increased his tidal air 108 per cent during convalescence as compared to the latter's 41 per cent.

Only 1 patient of the series exhibited very strikingly rapid and shallow breathing. This was case 13 whose tidal air was only $127 \mathrm{cc}$. and whose rate in the plethysmograph was 70 to the minute. In spite of the fact that his pulmonary minute volume was more than 100 per cent larger during the acute illness than it was subsequently during convalescence his arterial oxygen saturation was only 79.4 per cent. In a case such as this one, when the tidal air probably does not exceed the subject's dead air space, and alveolar ventilation must depend upon diffusion alone, it is reasonable to suppose that oxygen want is aggravated by the restricted character of the breathing.

Direct evidence for this may be had from observing the effect of morphine on some pneumonia patients (14) in whom a depression in respiration increases the already existing oxygen want. Case 17 may serve as an example of this, for after receiving $16 \mathrm{mgm}$. of morphine sulphate subcutaneously the patient's tidal air decreased from 331 to $254 \mathrm{cc}$., the rate from 32 to 25 and the minute volume from 10.67 liters to 6.38 liters. These changes were accompanied by a decrease of the oxygen content of the arterial blood from 13.05 vols. per cent to 11.47 vols. per cent and a reduction in saturation from 92.1 to 80.8 per cent. It should be borne in mind, however, that the depressed respiratory function resulting from morphine is of a different nature from that typical of pneumonia which is characterized by a rapid rather than a slow rate and an augmented rather than a diminished minute volume of pulmonary ventilation. 


\section{The effect of oxygen inhalation on the respiratory rate and depth}

Three patients of the series were studied both while breathing room air and again with the plethysmograph placed inside the oxgen chamber while the patients were permitted to inhale a 40 to 44 per cent oxygen mixture. It was hoped to glean from this procedure further information as to the relative interdependence of rapid and shallow breathing and anoxemia.

Case 14. J. G., male, age 23. Hospital No. 6357. Diagnosis: Lobar pneumonia; empyema; thoracotomy.

Present illness. For 2 days the patient had complained of pain in his left side and chills. He was admitted on March 18, 1928.

Physical examination. Severe pain was obvious. There was slight cyanosis of nail beds and face. The temperature was $105.4^{\circ} \mathrm{F}$., pulse 132 , respirations 60 . The leucotye count was 34,700. Examination of the chest showed slight dulness over angle of scapula. Below this point breath sounds were suppressed.

$X$-ray examination. Diffuse opacity from left apex to base, most marked at base.

Bacteriological examination. Group IV pneumococcus and hemolytic streptococcus were recovered from sputum. Blood culture sterile.

Course. Plethysmographic tracings were made before and during oxygen administration. This was accomplished by placing the plethysmograph inside the oxygen chamber. The first plethysmographic tracing was made March 19, a day after admission. The temperature was then $103.2^{\circ} \mathrm{F}$., pulse 128 . The respiratory rate was 44 , tidal air $255 \mathrm{cc}$., and minute volume 11.20 liters. The expiratoryinspiratory ratio was 0.96 . The arterial blood analysis showed a percentage saturation of 81.7 , with the oxygen content and capacity 16.15 and 19.79 vols. per cent, respectively. After these observations had been made the oxygen concentration in the chamber was raised to $\mathbf{4 4 . 5}$ per cent. Two hours later a second series of plethysmographic observations were made. The respiratory rate was now 46.7, tidal air $228 \mathrm{cc}$., and minute volume 10.67 liters. The ratio of expiration to inspiration was now 1.34. Arterial saturation had increased to 94.4 per cent with an oxygen content of 20.12 vols. per cent and capacity of 21.68 vols. per cent.

\section{Comment on case 14}

The result of putting the patient in the chamber was to raise the $\mathrm{O}_{2}$ content of the arterial blood approximately 4 vols. per cent and to increase the per cent saturation from 81.7 to 94.4 . No slowing or deepening of respirations occurred. It may be assumed that the 
anoxemia was not responsible, in this instance, for the presence of rapid and shallow breathing. We have frequently observed in the therapeutic use of oxygen that arterial anoxemia may be relieved with little or no effect on the character or rate of respirations.

Case 15. C. C., male, age 16. Hospital No. 6410. Diagnosis: Lobar pneumonia; focal nephritis.

Present illness. A young Italian boy was admitted to the hospital on April 17 complaining of pain in his left side and cough with expectoration of blood tinged sputum. He had been sick for 2 days.

Physical examination. The patient was slightly cyanosed. Temperature was $101.4^{\circ} \mathrm{F}$., pulse 128 , respirations 28 . The leucocyte count was 5,200 . The chest findings showed dulness and diminished voice sounds in the right front chest from the second rib to the base. Occasional crepitant râle heard. In the region of the spine of the right scapula breathing was broncho-vesicular. Many subcrepitant râles at right base. Dulness and suppressed breathing from second rib to base in left back.

$X$-ray examination. There was opacity of the right middle and lower lobes and upper part of the left lower lobe.

Bacteriological examination. No type specific organism was recovered from the sputum. Blood culture was sterile.

Course. On April 17 the patient was transferred to the oxygen chamber. Before the oxygen was turned on a plethysmographic record was obtained. At that time the patient's temperature was $102.4^{\circ} \mathrm{F}$., his pulse rate 144 . He was breathing at the rate of 46 to the minute. Tidal air was $266 \mathrm{cc}$. and minute volume 12.23 liters. The duration of the inspiratory phase was 0.63 second, of the expiratory 0.74 second. The ratio was 1.17 . The arterial blood was 65.8 per cent saturated, oxygen content 12.48 vols. per cent, capacity 18.97 vols. per cent.

Two and a half hours later, after the patient had been in an atmosphere of 40 per cent oxygen for more than an hour a second plethysmographic record was made. The respiratory rate was now 42.5 and the tidal air $236 \mathrm{cc}$. The minute volume 10.03 liters. No significant change in the duration of inspiration and expiration occurred. The saturation of the arterial-blood had fallen slightly, to 64.1 per cent. Oxygen content and capacity were respectively 11.23 and 17.50 vols. per cent. The patient grew rapidly worse and died on the following day.

\section{Comment on case 15}

The observations bring out one important point, namely, that exposing the patient to twice the normal atmospheric oxygen tension produced no changes in the oxygen saturation of the arterial blood and no change in the breathing. Were the anoxemia due to rapid and shallow breathing, it is logical to assume that raising the alveolar oxy- 
gen tension two-fold would, in a measure at least, relieve the condition. In this instance we must assume that blood was circulating through unaerated portions of the lungs, and that this rather than rapid and shallow breathing was in the main responsible for oxygen want.

Case 16. J. M., male, age 32. Hospital No. 6414. Diagnosis: Lobar pneumonia; septicemia.

Present illness. The patient had been sick for 3 days, complaining of pain in the left chest, chills, cough, and expectoration of blood tinged sputum. He was brought to the hospital on April 19,1928.

Physical examination. There was slight cyanosis of nail beds. Temperature was $104^{\circ} \mathrm{F}$., pulse 120 , respirations 36 . The leucocyte count was 14,000 . The chest signs showed; from spine of left scapula to base posteriorly there was dulness to flatness; voice and breath sounds increased except at very base where they were diminished; bronchial breathing and occasional crepitant râle at angle of left scapula; dulness and occasional sub-crepitant râle at the right base.

$X$-ray examination. Opacity of area occupied by left lower lobe, lower part of right upper lobe and right lower lobe.

Bacteriological examination. Group IV pneumococcus was recovered from the sputum and blood.

Course. On April 20 the patient was transferred to the oxygen chamber because he was growing more cyanotic and his breathing was becoming more difficult. Before oxygen was administered a plethysmographic record was made. His temperature was then $102.8^{\circ} \mathrm{F}$., pulse 112 . The respiratory rate was 35 , tidal air $376 \mathrm{cc}$., and minute volume 13.18 liters. The arterial blood oxygen content was 18.05 vols. per cent, with a capacity of 20.60 vols. per cent, the saturation being 87.7 per cent.

Two and a half hours later, after the patient had been in an atmosphere of 39.5 per cent oxygen for $1 \frac{1}{2}$ hours, a second plethysmographic tracing was made. No significant change had occurred in any of the measurements. The respiratory rate was now 35.2, tidal air $372 \mathrm{cc}$., minute volume 13.08 liters. The arterial oxygen content and capacity had risen respectively to 18.54 and 20.80 vols. per cent. The saturation was now 89.0 per-cent. In spite of this slight increase the patient's color was obviously better and he said he felt better.

The next day the patient became rapidly worse and died on April 23. His blood culture was positive, there being more than 100 colonies to the cubjc centimeter of blood.

\section{Comment on case 16}

The case is similar to the last one, a rapidly fatal pneumonia which did not respond to oxygen therapy either by a significant increase in the per cent saturation of arterial blood or by a slowing or deepening 
of the respirations. Were the anoxemia the result of rapid and shallow breathing, one would expect relief from raising the atmospheric oxygen tension, especially in a case where neither rapid rate nor shallow breaths were extreme.

\section{DISCUSSION}

There can be no doubt of the frequent association of anoxemia and rapid and shallow breathing in pneumonia. Reference to table 1 will provide numerous examples of this fact. That these two phenomena, however, are causally and reciprocally related does not necessarily follow. In a paper dealing with this subject Meakins (6) concluded "that the anoxemia occurring in acute lobar pneumonia is the result of the rapid and shallow breathing typical of this condition." This statement is based on 4 published cases in which no blood analyses are included. Our observations do not wholly support this contention, since exceptional cases occur in which no correlation between changes in the rate and depth of breathing and the oxygen saturation of the arterial blood can be demonstrated (cases 7 and 11). There are, of course, individuals whose pulmonary ventilation is so inadequate (case 13) that it must self evidently lead to asphyxia; and others in whom a reduction in ventilation following morphine injection will accentuate already existing oxygen want (case 3). Two cases (nos. 15 and 16) both anoxemic while breathing room air remained so in an atmosphere containing twice the normal partial pressure of oxygen. Were anoxemia in these cases the result of rapid, shallow breathing there should have been an alleviation of oxygen want, which did not, however, come to pass. The fact that the blood may be restored to its normal oxygen content without a reduction in respiratory rate or an increase in the depth of breathing (case 14) is strong evidence against the corollary view that rapid and shallow breathing in pneumonia is the result of anoxemia.

It is likely that in this physiological function, as in others, reduction beyond a threshold value is necessary before the internal environment of the body suffers appreciable variation. In other words, there is a factor of safety beyond which ventilation must be reduced before anoxemia arises. There is no convincing evidence to prove that the anoxemia commonly occurring in pneumonia arises from such a reduc- 
tion, though it is reasonable to believe that in certain instances it may be an aggravating influence.

How much actual acceleration in rate irrespective of diminished depth has to do with inadequate reoxygenation of blood in the lungs has not, heretofore, been considered. The shortening of the duration of the inspiratory and expiratory phases and the reduction in the expiratory-inspiratory ratio was, in these patients, the rule during their acute illness. The significance of this change is not clear. We have the impression, however, that the relative duration of the respiratory phases is of importance from the point of view of pulmonary ventilation.

Relatively large values for the minute volume of pulmonary ventilation are found almost without exception during the acute stage of pneumonia, and become smaller during convalescence. This has been looked upon by some as of a compensatory nature. If so, the compensation is obviously inadequate with respect to the aeration of the blood in the lungs.

With regard to the vital capacity, its measurement is perhaps of not much significance except as an indication of pleuritic pain. The functional residual air studied by Binger and Brow (7) is of greater interest, and this we believe should be reinvestigated from the point of view of anoxemia.

\section{SUMMARY AND CONCLUSIONS}

1. By means of a specially designed body plethysmograph the respiratory movements have been studied in a group of patients suffering from acute pneumonia.

2. The oxygen content, capacity and per cent saturation have also been measured.

3. Observations have been made during the acute stage of the disease, during convalescence, after morphine administration and oxygen inhalation.

4. Rapid and shallow breathing, and anoxemia are commonly associated phenomena.

5. No clear evidence can be adduced, however, that the anoxemia which occurs in lobar pneumonia is the result of rapid and shallow breathing, though in some cases extremely rapid and shallow breath- 
ing or the depression of ventilation brought on by morphine may accentuate already existing oxygen want.

6. Oxygen inhalation may restore the arterial blood to a normal saturation without influencing pulmonary ventilation.

\section{BIBLIOGRAPHY}

1. Binger, Carl A. L., Hastings, A. Baird, and Sendroy, Julius, Jr., J. Exp. Med., 1927, xlv, 1081. A Further Study of Blood Reaction and Blood Gases in Pneumonia.

2. Binger, Carl A. L., J. Clin. Invest., 1928, vi, 203. Anoxemia in Pneumonia and Its Relief by Oxygen Inhalation.

3. Haldane, J. S., Respiration, New Haven, 1922, p. 137.

4. Lundsgaard, Christen, and Van Slyke, Donald D., Cyanosis, Baltimore, 1922. Medicine Monographs, Vol. II.

5. Haldane, J. S., Meakins, J. C., and Priestly, J. G., J. Physiol., 1918-1919, lii, 433. The Effects of Shallow Breathing.

6. Meakins, J. C., Arch. Int. Med., 1920, xxv, 1. Harmful Effects of Shallow Breathing with Special Reference to Pneumonia.

7. Binger, Carl A. L., and Brow, George R., J. Exp. Med., 1924, xxxix, 677. Studies on the Respiratory Mechanism in Lobar Pneumonia; A Study of Lung Volume in Relation to the Clinical Course of the Disease.

8. Hoover, C. F., J. Am. Med. Assn., 1918, lxxi, 880. Moisture in the Air Spaces of the Lungs and Oxygen Therapy.

9. Schjerning, J., Beitr. z. Klin. d. Tuberc., 1922, 1, 96 . Utber das Problem der Zyanose und den Begriff der Pneumonose.

10. Stadie, Wm. C., J. Exp. Med., 1922, xxxv, 337. The Treatment of Anoxemia in Pneumonia in an Oxygen Chamber.

11. Binger, C. A. L., Brow, G. R., and Branch, Arnold, J. Clin. Invest., 1924, i, 155. Experimental Studies on Rapid Breathing. II. Tachypnea Dependent upon Anoxemia Resulting from Multiple Emboli in the Larger Branches of the Pulmonary Artery.

12. Binger, Carl A. L., and Davis, John S., Jr., Proc. Soc. Exp. Biol. and Med., 1928, xxv, 607. A Body Plethysmograph for the Study of Respiratory Movements in Human Beings.

13. Van Slyke, D. D., and Neill, J. M., J. Biol. Chem., 1924, lxi, 523. The Determination of Gases in Blood and Other Solutions by Vacuum Extraction and Manometric Measurement.

14. Davis, John' S., Jr., J. Clin. Invest., 1928, vi, 187. The Effect of Morphine on the Respiration in Pneumonia. 